\title{
THE STRUCTURE OF LOCALLY CONNECTED TOPOLOGICAL SPACES
}

\author{
BY \\ G. E. ALBERT AND J. W. T. YOUNGS \\ INTRODUCTION
}

0.1. This paper presents an investigation of the following problem. Exhibit a class $X$ of topological spaces which contains all peano spaces and which has the following properties: (1) a cyclic element theory exists in each space of the class, (2) the abstract set consisting of all cyclic element of any space $X$ of the class can be topologized so as to be a member of the class $X$, and (3) the hyperspace thus obtained is acyclic. Since the class $P$ of all peano spaces does not satisfy the condition (2), it is clear that any solution of the problem lies in a generalization of peano spaces. (For cyclic element theory, see Whyburn [5]( $\left.{ }^{1}\right)$, and [6], or Kuratowski and Whyburn [4].)

One such generalization has been proposed by R. L. Moore [5]; another by one of the authors (Youngs [9]). Moore employed two primitive concepts: region and contiguity (compare this with satelliticity 4.8). Youngs used the notion of arc as primitive. In the following pages a solution is given which is based upon the usual concept of open set.

0.2. The work is divided into four sections. The first of these is devoted to the definition and a brief discussion of the class $X$ of spaces to be used in the remainder of the paper; namely, locally connected topological spaces. No separation or countability properties are assumed. Thus, in particular, a single point need not form a closed point set. The use of such a weak topology is not dictated merely by a desire for generality; indeed, it is shown in later sections that this weakness is fundamental in the consideration of hyperspaces of peano spaces.

The development of a theory of cyclic elements for spaces of the class $X$ occupies the second section. In such spaces, the standard definitions of "cut point" and "cyclic element" (Kuratowski and Whyburn [4]) fail to yield certain important properties of these concepts. However, the properties are easily recovered by generalizations of the definitions mentioned. The degree of similarity achieved between the cyclic structure of spaces of the general class $X$ and of peano spaces seems remarkable in view of the weak topology assumed in the former.

Presented to the Society in part on April 12, 1940 by the first of the authors under the title On contiguous point spaces and their applications, and in final form under the present title on April 12, 1941; received by the editors April 30, 1941.

(1) Numbers in brackets refer to the bibliography. 
The third section of the paper contains a discussion of the hyperspace of cyclic elements of any locally connected topological space. In particular, it is shown that the class of all such spaces solves the problem stated in 0.1. Moreover, it is shown that the hyperspace can always be defined as the strongly continuous image (see 3.4 ) of the original space.

0.3. It is easily seen that the solution offered for the problem of 0.1 is not unique. The concluding section of the paper is devoted to a discussion of certain subclasses of $\mathcal{X}$ which also solve the problem. In this study a new concept, that of hereditary classes of spaces, arises naturally. Briefly, a subclass $\mathfrak{H C}$ of $X$ is called hereditary if, whenever $X$ is in $\mathcal{H C}$, the hyperspace of $X$ is in $\mathfrak{H C}$ and every true cyclic element of $X$ is a member of $\mathfrak{F}$. The class $\mathcal{P}$ of all peano spaces is not hereditary. However, $X$ is a hereditary class that contains $P$. It is interesting to note that there are highly restricted hereditary subclasses of $\mathcal{X}$ which contain the class $P$. For example, one such subclass is composed of all the locally connected topological spaces which satisfy the $T_{0}$ separation axiom, and which are, in addition, strongly continuous images of the closed unit interval of the number axis. This class of spaces yields considerable insight into the nature of the hyperspaces of peano spaces.

\section{The SPACE $X$}

1.1. The symbol $X$ will denote a class of elements (the space); elements of $X$, to be called points, will be denoted by small Latin letters; point sets will be designated by capital Latin letters. The usual logical concepts and notations will be employed in dealing with point sets.

It will be supposed that there is defined in $X$ a definite class $\mathfrak{O}$ of point sets. A set will be called open if and only if it belongs to $\mathfrak{D}$. The collection $X$ is called a topological space if:

A1. The class $X$ and the empty set are open.

A2. The product of any two open sets is open.

A3. The sum of any number of open sets is open.

Only spaces of this character will be considered.

1.2. A point set will be called closed if it is the complement of an open set.

1.3. If $A$ is a point set in $X$, the symbol $\bar{A}$ will denote the product of all the closed subsets of $X$ that contain $A$. The set $\bar{A}$ will be called the closure of the set $A$.

1.4. A space in which the closure $\bar{A}$ of an arbitrary set $A$ is the primitive concept is called a Kuratowski space whenever the closure function satisfies the axioms:

$$
\overline{A+B}=\bar{A}+\bar{B}, \quad A \subset \bar{A}, \quad \overline{\bar{A}} \subset \bar{A}, \quad \bar{O}=0 .
$$

A topological space is completely equivalent to a Kuratowski space. The proof of this theorem is well known (Alexandroff and Hopf [1, p. 41] or Hausdorff 
$[2$, p. 227 ]) and will not be reproduced here. The following consequence of the result will be used in the sequel without further mention.

If $p$ is a point and $A$ is a set, then $p \in \bar{A}$ if and only if every open set that contains $p$ intersects $A$.

1.5. The symbol $F(A)$ will denote the set

$$
\bar{A} \cdot(\overline{X-A})
$$

which is called the frontier of the set $A$. It is easily seen that the frontier of any set is closed.

1.6. A point set $E$ will be called connected if it cannot be decomposed into two nonvacuous sets $A$ and $B$ such that $A \cdot \bar{B}=0=\bar{A} \cdot B$. Any degenerate set (the empty set or any set consisting of a single point) is connected. If $E$ is connected and $E \subset C \subset \bar{E}$, then $C$ is connected. (For other results on connected sets in general spaces see Knaster and Kuratowski [3].)

1.7. If $S \neq 0$ is a maximal connected subset of $E$, then $S$ is called a component of $E$.

1.8. The topological space $X$ will be called locally connected if each component of any open set is open. This definition is equivalent to the usual one in peano spaces.

In the remainder of the paper it will be assumed that $X$ is a locally connected topological space.

1.9. Lemma. If $G_{1}$ and $G_{2}$ are open, disjoint point sets, then $G_{1} \cdot \bar{G}_{2}=0=\bar{G}_{1} \cdot G_{2}$. and $\bar{G}_{1} \cdot \bar{G}_{2} \subset X-\left(G_{1}+G_{2}\right)$.

1.10. THEOREM. If $E$ is a closed point set and $S$ is any component of the set $X-E$, then $F(S) \subset E$.

Proof. Let $R$ be the sum of all the components of $X-E$ which are distinct from $S$. Since $S$ is open $F(S) \subset \overline{X-S}=X-S$. On the other hand, $F(S) \subset \bar{S}$ and $\bar{S} R=0$ since $S$ is a component of $S+R$. Therefore, $F(S) \subset X-S-R=E$.

\section{Cyclic elements}

2.1. In this section a definition will be given for a class of subsets of the space $X$ which will be called cyclic elements. The concept as introduced here is easily shown to reduce to the familiar one in case $X$ is a peano space.

The structure of a locally connected topological space will depend only upon the structure of its individual components. Thus, there will be no loss in generality in assuming that the space $X$ is connected and this will be done. The restriction will be removed in 3.18 .

2.2. A point $p$ will be said to separate two points $a$ and $b$ if: (1) $p=p$, and (2) the points $a$ and $b$ lie in distinct components of the set $X-p$. Any point of this character will be called a cut point of the space $X$.

The exclusion of all points which are not closed from the class of cut points 
is not an artificial condition. The reader will recall that in peano spaces the essential tool in the use of cut points is the property that the components of the complement of any cut point are open sets. This property would be lost here without the condition (1) on cut points.

2.3. Two points $a$ and $b$ will be called conjugate (denoted by $a \sim b$ ) if they are separated by no point of $X$. Conjugacy is reflexive and symmetric.

Note that, as the term separate does not apply to a point $p$ such that $p \neq p$, two points $a$ and $b$ may be conjugate even though they lie in distinct components of the set $X-p$.

2.4. Lemma. If $a \sim x_{1} \sim \cdots \sim x_{n} \sim b$, and the point $z$ separates $a$ from $b$, then $z=x_{i}$ for some $i=1, \cdots, n\left({ }^{2}\right)$.

Proof. If the statement is false, then all the points $a, x_{1}, \cdots, x_{n}, b$ lie in a single component of the set $X-z$.

2.5. Corollary. If $a \sim x \sim b$ and $\bar{x} \neq x$, then $a \sim b$.

2.6. Corollary. If $a \sim x_{1} \sim \cdots \sim x_{n} \sim b, a \sim y_{1} \sim \cdots \sim y_{m} \sim b$ and all the points $x_{1}, \cdots, x_{n}, y_{1}, \cdots, y_{m}$ are distinct, then $a \sim b$.

2.7. A point set $E$ will be called coherent if for every pair of points $a$ and $b$ contained in $E$ it is true that $a \sim b\left({ }^{2}\right)$.

2.8. A point set $E$ will be called complete if $E$ contains every point $z$ which is conjugate to each of two distinct points contained in $E\left({ }^{2}\right)$.

2.9. Lemma. If $E$ is any coherent set and $x$ is any point such that $\bar{x}=x$, then the set $E-x$ is contained in a single component of the set $X-x$.

2.10. A point set $N$ which is nondegenerate, complete, and coherent will be called an $N$-set.

2.11. Theorem. If $a \sim b$ and $a \neq b$, then there exists $a$ unique $N$-set containing $a$ and $b$.

Proof. Denote by $N$ the totality of points which are conjugate to both $a$ and $b$. If $x$ and $y$ are any pair of distinct points in the set $N$, then by 2.6, $x \sim y$. Thus $N$ is a coherent set. Suppose that $z$ is a point conjugate to both $x$ and $y$. Since $x \sim a \sim y$, it follows by 2.6 that $z \sim a$. Similarly, $z \sim b$. Thus $z$ is in the set $N$, and $N$ is a complete set. By definition $N$ is an $N$-set.

If $N^{\prime}$ is any other $N$-set containing $a+b$, it follows directly from the completeness of the sets $N$ and $N^{\prime}$ that they are identical.

2.12. TheOREM. If $N_{1}$ and $N_{2}$ are distinct $N$-sets, then $N_{1} \cdot N_{2}$ is either vacuous or a single point $x$ which separates any point of $N_{1}-x$ from every point of $N_{2}-x$.

(2) The importance of Lemma 2.4 and the definitions in 2.7 and 2.8 were noticed first by Radó and Reichelderfer [8]. 
Proof. By 2.11, the product $N_{1} \cdot N_{2}$ is either vacuous or a point $x$. Let $a_{i} \neq x$ be in $N_{i}$ for $i=1,2$. If $\bar{x} \neq x$, then by $2.5, a_{1} \sim a_{2}$. If $\bar{x}=x$ but $x$ does not separate $a_{1}$ from $a_{2}$, then by $2.4, a_{1} \sim a_{2}$. In either case $a_{1} \sim a_{2} \sim x$ and thus by the completeness of $N_{1}$ it contains $a_{2}$. This contradicts 2.11 .

\subsection{Theorem. Any $\mathrm{N}$-set is closed.}

Proof. Suppose that the point $p \in \bar{N}$. Choose any point $a \in N$. If there is a point $x$ such that $\bar{x}=x$ and $a \neq x \neq p$, let $S$ be the component of $X-x$ containing $p$. Now $S$ is open by 1.8 , and so contains a point of the set $N$. As $N$ is coherent, $S$ contains the point $a$. Thus $p \sim a$. If no such point $x$ exists, $p \sim a$ by the definition of 2.2. But the point $a$ was arbitrarily chosen in $N$. Since $N$ is nondegenerate and complete, $p \in N$.

Remark. In connection with the remarks of 2.2 , the theorem of the present section would be false without the generalized definition of cut points introduced in 2.2 .

2.14. Consider any $N$-set, $N$. Define the subset $k(N)$ of $N$ by: the point $x$ is in $k(N)$ if and only if (1) $x \in N$, and (2) for no component $S$ of the set $X-N$ is it true that $x \in F(S)$. The set $k(N)$ will be called the kernel of the set $N$.

Clearly, by $1.10, N=k(N)+\sum F(S)$ where the summation extends over all components $S$ of the set $X-N$.

2.15. A subset $M$ of the space $X$ will be termed a true cyclic element if: (1) it is an $N$-set, (2) the set $S$ being any component of $X-M$, the frontier $F(S)$ is a single point, and (3) the kernel $k(M)$ is nondegenerate. The symbol $M$ will be employed as a generic notation for true cyclic element.

2.16. If the space $X$ is peanian, it can be shown that every $N$-set given by the definition of 2.10 is a true cyclic element under the definition of 2.15. Moreover, since every point in a peano space is a closed set, the definitions of cut point and conjugate points given in 2.2 and 2.3 reduce to the standard notions for such spaces. It follows readily that, for peano spaces, the definition of true cyclic elements given here reduces to the usual definition (Kuratowski and Whyburn $[4])$.

2.17. A point which is not a cut point of the space and which is contained in no true cyclic element will be called singular. Cut points and singular points will be termed degenerate cyclic elements. It follows immediately that:

2.18. ThEOREM. The cyclic elements of the space $X$ cover it.

2.19. TheOREM. If $M$ is a true cyclic element and $x$ is a point of $M$, then $x \in M-k(M)$ if and only if $x$ is a cut point of the sface $X$.

Proof. Suppose $x$ is a cut point of $X$. Using 2.9 select a component $S$ of $X-x$ such that $S \cdot M=0$. Then $S$ is also a component of $X-M$. But $x=F(S)$, so $x \in M-k(M)$.

Suppose $x \in M-k(M)$. There exists a component $S$ of $X-M$ such that 
$x=F(S)$. If $R$ denotes the sum of all the remaining components of $X-M$, it is easy to show by 1.9 and 1.10 that

$$
S \cdot[\overline{(M-x)+R}]=0=\bar{S} \cdot[(M-x)+R]
$$

and since $X-x=[(M-x)+R]+S$, the lemma follows.

2.20. Corollary. If $M$ is a true cyclic element and $S$ is any component of $X-M$, then the point $x=F(S)$ separates any point in $S$ from every point in $M-x$.

2.21. Lemma. If $E$ is any closed set, then

$$
F\left(\sum S\right)=\overline{\sum F(S)}
$$

where the summation extends over any class of components $S$ of $X-E$.

Proof. Clearly $\sum F(S) \subset F\left(\sum S\right)$, and as the frontier of any set is closed (see 1.5),

$$
\overline{\sum F(S)} \subset F\left(\sum S\right)
$$

Suppose $p \in F\left(\sum S\right)$ and $p \in X-\overline{\sum F(S)}$. Consider $U$, the component of $X-\overline{\sum F(S)}$ containing $p$. For some term $S$ of $\sum S, U \cdot S \neq 0$. Now $U=U \cdot S$ $+(U-S)$. Since $U \cdot S$ is open $(1.8),(U \cdot S) \cdot(\overline{U-S})=0$. Also $(U-S) \cdot(\overline{U \cdot S})$ $=(U-S) \cdot[U \cdot S+F(U \cdot S)]=(U-S) \cdot F(U \cdot S) \subset(U-S) \cdot[F(U)+F(S)]$ $=(U-S) \cdot F(S)=0$. This contradicts the connectedness of the set $U(1.6)$. Thus $F\left(\sum S\right) \subset \overline{\sum F(S)}$.

2.22. If $M$ is a true cyclic element and if $E$ is a subset of $M$; then $E^{*}$ will denote the set $E+\sum S$ where the summation is taken over all components $S$ of $X-M$ such that $F(S) \in E$.

2.23. Lemma. If $M$ is a true cyclic element and the set $E$ is closed in $M$, then the $E^{*}$ is closed in $X$.

Proof.

$$
\begin{aligned}
\overline{E^{*}} & =\overline{E+\sum S}=\bar{E}+\overline{\sum S}=E+\sum S+F\left(\sum S\right) \\
& =E+\overline{\sum F(S)}+\sum S=E+\sum S=E^{*} .
\end{aligned}
$$

The various equalities follow easily from 2.21 and the fact that $E$ is closed in $X$.

2.24. Corollary. If $M$ is a true cyclic element and the set $E$ is open in $M$, then $E^{*}$ is open in $X$.

2.25. Lemma. If the set $E$ is connected in $M$, then $E^{*}$ is connected in $X$.

The lemma follows at once from the fact that if $E$ is connected in $M$ it is connected in $X$ by 1.6 . 
2.26. Lemma. If $A$ and $B$ are subsets of some true cyclic element $M$ and $A \cdot \bar{B}=0$, then $A^{*} \bar{B}^{*}=0$.

Proof. If $\sum_{A} S$ and $\sum_{B} S$ denote the sums of all components of $X-M$ whose frontiers are in $A$ and $B$ respectively, then by 2.21

$$
\begin{aligned}
A^{*} \bar{B}^{*}= & \left(A+\sum_{A} S\right) \cdot\left(\overline{B+\sum_{B} S}\right) \\
= & \left(A+\sum_{A} S\right) \cdot\left[\left(B+\sum_{B} S\right)+F\left(B+\sum_{B} S\right)\right] \\
= & \left(A+\sum_{A} S\right) \cdot F\left(B+\sum_{B} S\right) \\
& C\left(A+\sum_{A} S\right) \cdot\left[F(B)+F\left(\sum_{B} S\right)\right] \\
= & \left(A+\sum_{A} S\right) \cdot\left[F(B)+\sum_{B} F(S)\right] \\
& \subset\left(A+\sum_{A} S\right) \cdot \bar{B} \subset\left(\sum_{A} S\right) \cdot \bar{B}=0,
\end{aligned}
$$

since $A \bar{B}=0$ and $\bar{B} \subset M \subset X-\sum_{A} S$.

2.27. Theorem. If $Z$ is a connected set and $M$ is a true cyclic element, then $M Z$ is connected.

Proof. If $M Z$ is degenerate, the theorem is obvious (1.6). Suppose $M Z=A+B$, where $A \neq 0 \neq B$ and $A \bar{B}=0=\bar{A} B$. Now if $p \in Z-M$, suppose that $S$ is the component of $X-M$ that contains $p$. Clearly $F(S) \subset M Z$, for otherwise $Z=(S Z)+(Z-S)$ would be a decomposition showing that $Z$ is not connected. Thus $p \in(M Z)^{*}$. Hence $Z \subset(M Z)^{*}=A^{*}+B^{*}$, and $A^{*} \bar{B}^{*}=0=\bar{A}^{*} B^{*}$ by 2.26 . On the other hand $Z A^{*} \neq 0 \neq Z B^{*}$; hence the connectedness of $Z$ is contradicted.

The validity of this theorem seems surprising in view of the fact that the proof given for peano spaces (Kuratowski and Whyburn [4]) depended upon the metric and the fact that the number of components of an open set is at most denumerable. The consequences of this product theorem are as varied and important here as in peano spaces.

2.28. Corollary. If $x$ is a point such that $\bar{x}=x$ and $M$ is a true cyclic element, then the set $M-x$ is connected.

The truth of this corollary follows from 2.9 and 2.27.

2.29. TheOREM. A true cyclic element is a connected, locally connected, topological space. 
Proof. The connectedness follows from 2.27 and the fact that $X$ is connected. Let $G$ be open in a cyclic element $M$. It will be shown that each component of $G$ is open in $M$. By 2.24 the set $G^{*}$ is open in $X$. Let $S^{*}$ be any component of $G^{*}$. By 2.27, $S^{*} M$ is connected and nonvacuous., Using 2.25, $S^{*} M$ is a component of $G$. But $S^{*}$ is open in $X$ by 1.8 ; hence $S^{*} M$ is open in $M$. Using 2.25 it is easy to see that all the components of $G$ are obtained in this manner.

Finally the subsets of $M$ which are open in $M$ clearly satisfy A1, A2 and A3 of 1.1.

\section{The hyPERSPACE}

3.1. Paralleling the notation in the space $X$, the symbol $X_{h}$ will be reserved for the abstract set composed of all cyclic elements (both true and degenerate) of the space $X$. Elements of the set $X_{h}$ will be denoted by small Greek letters; sets of elements in $X_{h}$ will be denoted by large Greek letters.

3.2. It follows easily from 2.12 and 2.17 that, if $x$ is any point of the space $X$, there exists a smallest cyclic element in $X$ which contains the point $x$. This fact makes possible the definition of a single-valued transformation $T(X)=X_{h}$ from any locally connected topological space $X$ to the class $X_{h}$ of its cyclic elements as follows: if $x$ is any point of the space $X$, then $T(x)=\xi$, where the element $\xi$ of $X_{h}$ is the smallest cyclic element of $X$ which contains the point $x$.

The following familiar conventions will be employed.

If $A$ is a subset of $X$, then $T(A)=E_{\xi}[\xi=T(x), x \in A]$ will be called the image of the set $A$.

If $\Delta$ is a subset of $X_{h}$, then $T^{-1}(\Delta)=E_{x}[T(x) \in \Delta]$ will be called the inverse of $\Delta$ in $X$. A set $D$ in $X$ will be called an inverse set if and only if there is some set $\Delta$ such that $T^{-1}(\Delta)=D$.

It is clear that the inverse of a single element of $X_{h}$ is either a degenerate cyclic element in $X$ or the kernel of some true cyclic element in $X$ (see 2.15).

3.3. The transformation, $T$, will now be used to topologize the set $X_{h}$ in accordance with the convention: a subset $\Gamma$ of $X_{h}$ will be called open if and only if its inverse, $T^{-1}(\Gamma)$, is an open set in the space $X$.

THEOREM. The class of open sets in $X_{h}$ satisfies the axioms A1, A2, and A3.

Proof. The theorem follows at once from the formulas $T^{-1}\left(\Gamma_{1} \cdot \Gamma_{2}\right)$ $=T^{-1}\left(\Gamma_{1}\right) \cdot T^{-1}\left(\Gamma_{2}\right)$, and $T^{-1}\left(\Sigma \Gamma_{\alpha}\right)=\Sigma T^{-1}\left(\Gamma_{\alpha}\right)$.

The set $X_{h}$ may now be thought of as a topological space. As such, it will be termed the hyperspace of $X$.

3.4. A transformation from one topological space to another is said to be continuous if the inverse of an open set is open. The transformation is said to be strongly continuous if, in addition, any open inverse set has an open image (Alexandroff and Hopf $[1$, p. 65]). 
3.5. THEOREM. The transformation $T(X)=X_{h}$ is strongly continuous under the topologization of $X_{h}$ given in 3.2, and this is the only topologization which will make $T$ strongly continuous. in $X$.

3.6. Lemma. $A$ set $\Phi$ is closed in $X_{h}$ if and only if the set $T^{-1}(\Phi)$ is closed

3.7. Lemma. If $E$ is connected in $X$ then $T(E)$ is connected in $X_{h}$.

The first of these lemmas is obvious; the second is well known.

3.8. It will be shown that the hyperspace $X_{h}$ is a locally connected topological space. The proof requires the

Lemma. If $\Delta$ is any set in $X_{h}$ and $\Sigma$ is a component of $\Delta$, then $T^{-1}(\Sigma)$ is the sum of certain components of the set $T^{-1}(\Delta)$.

Proof. Let $x \in T^{-1}(\Sigma)$ and let $S$ denote the component of $T^{-1}(\Delta)$ which contains $x$. Now $T(S)$ is connected by 3.7 and intersects $\Sigma$. Thus $T(S) \subset \Sigma$. Hence $S \subset T^{-1}(\Sigma)$ and the lemma follows.

Theorem. The space $X_{h}$ is locally connected.

Proof. If $\Gamma$ is open in $X_{h}$ and $\Sigma$ is a component of $\Gamma$, then $T^{-1}(\Sigma)$ is the sum of certain components of $T^{-1}(\Gamma)$. But, as $T$ is continuous, the set $T^{-1}(\Gamma)$ is open; since $X$ is locally connected, each component of $T^{-1}(\Gamma)$ is open. Therefore $T^{-1}(\Sigma)$ is open by A3. But $T^{-1}(\Sigma)$ is an inverse set so $\Sigma=T\left(T^{-1}(\Sigma)\right)$ is open by the strong continuity of $T$.

3.9. Consider the results achieved so far. If $X$ is any locally connected topological space, then by 2.29 , so is each true cyclic element of $X$; and, by 3.3 and 3.8, so is the hyperspace $X_{h}$. Thus a type of permanence of form is exhibited by the class of all locally connected topological spaces. This remark will furnish the basis of the discussion of the final section of the paper ( $\$ I V)$. It has also been shown that the hyperspace $X_{h}$ is always related to the original space $X$ by a strongly continuous, single-valued transformation, and that, relative to this property, the topology in $X_{h}$ is uniquely determined.

To complete the solution of the problem proposed in 0.1 it remains only to show that the hyperspace $X_{h}$ is always acyclic. The proof of this result seems to be difficult. It will be accomplished through a sequence of lemmas of which the first four are concerned with the components of certain sets in $X_{h}$, and the remainder deal with the relationship of conjugacy in $X_{h}$.

3.10. Lemma. A subset $E$ of the space $X$ is an inverse set if and only if, $M$ being any true cyclic element, $E \cdot k(M) \neq 0$ implies $E \supset k(M)$.

The proof is obvious (3.2).

3.11. Lemma. If $E$ is an inverse set of $X$ such that for any true cyclic element $M$ whose kernel is not in $E$ the set $E \cdot M$ is degenerate, then any component 
$S$ of $X-E$ is an inverse set, and if $E$ is closed, then $T(S)$ is a component of $X_{h}-T(E)$.

Proof. Consider any component $S$ of $X-E$. Let $M$ be any true cyclic element such that $k(M) \cdot S \neq 0$. Then $E \cdot k(M)=0$ by 3.10. If $E \cdot M=0$, then $S \supset M$ as $M$ is connected (2.29). If $E \cdot M=x$, then $x \notin k(M)$ and $\bar{x}=x(2.19)$. Hence $M-x$ is connected (2.28), and lies in $X-E$. Therefore, $k(M) \subset M$ $-x \subset S$. Hence, by 3.10 , the set $S$ is an inverse set.

Now suppose $E$ is closed. Let $R$ denote the sum of all the components of $X-E$ distinct from $S$. As above, $R$ is a sum of inverse sets and so is an inverse set. Moreover, $S$ and $R$ are open; hence $T(S)$ and $T(R)$ are open and have no points in common (3.2). Therefore, $T(S) \cdot \overline{T(R)}=0=\overline{T(S)} \cdot T(R)$ by 1.9. Now $T(S) \subset X_{h}-T(E)$ and is connected (3.7). Therefore $T(S)$ is a component of $X_{h}-T(E)$.

Corollary. If $M$ is a true cyclic element and $S$ is a component of $X-M$, then $S$ is an inverse set and $T(S)$ is a component of $X_{h}-T(M)$.

3.12. Lemma. With the hypotheses of 3.11, every component $\Sigma$ of $X_{h}-T(E)$ is the image of some component $S$ of $X-E$.

Proof. The set $T^{-1}(\Sigma)$ is a sum of certain components of $X-E(3.8)$. By 3.11 , each of these components is an inverse set and their images are components of $X_{h}-T(E)$. However, these images all intersect $\Sigma$ and therefore are identical to $\Sigma$.

3.13. Lemma. If $E$ is a subset of a true cyclic element $M$ and $S$ is a component of $X-M$ whose frontier is in $E$, then $S$ is a component of $X-E$.

Proof. Consider any $A \supset S$ such that $A \subset X-E$. Let $B=A-S$. Now $\bar{B} \cdot S=0$ and $\bar{S} \cdot B=[S+F(S)] \cdot B=B \cdot F(S)=0$, as $F(S) \in E$ and $B C X-E$. Hence $S$ is a component of $X-E$.

3.14. Lemma. If $M$ is a true cyclic element in $X$ and if $a \in M-k(M)$ and $\alpha=T(a), \beta=T(k(m))$; then $\alpha \sim \beta$.

Proof. Suppose that $\alpha$ is not conjugate to $\beta$; then there is some point $\xi=\bar{\xi}$ in $X_{h}$ such that $\xi$ separates $\alpha$ from $\beta$. Let $b \in k(M)$. Then $a+b \subset X-T^{-1}(\xi)$ and the components of $X-T^{-1}(\xi)$ that contain $a$ and $b$, respectively, must be distinct (3.7). Now $T^{-1}(\xi)$ cannot be a single point for if it were it would separate $a$ and $b$, denying the fact that $a \sim b$ as $M$ is coherent.

Then $T^{-1}(\xi)=k\left(M_{0}\right)$ for some true cyclic element $M_{0}$, and $M \neq M_{0}$ since $\xi \neq \beta$ and $T^{-1}(\beta)=k(M)$. Thus $a+b \subset M \subset X-T^{-1}(\xi)$. But $M$ is connected (2.29) and this contradicts the fact that the components of $X-T^{-1}(\xi)$ which contain $a$ and $b$ are distinct.

3.15. Lemma. If $\alpha$ and $\beta$ are distinct conjugate points in $X_{h}$, then either (1) 
one of the sets $T^{-1}(\alpha), T^{-1}(\beta)$ is the kernel, $k(M)$, of some true cyclic element $M$ and the other is a single point in $M-k(M)$; or (2) both $T^{-1}(\alpha)$ and $T^{-1}(\beta)$ are single points which are conjugate.

Proof. The proof is left to the reader. It follows easily from the fact that if the two inverse sets $T^{-1}(\alpha)$ and $T^{-1}(\beta)$ are separated in $X$ by a point $x$ (which is a closed inverse set in $X$ ), then $\alpha$ is separated from $\beta$ by $T(x)$ in $X_{h}$ (see 3.11).

3.16. Lemma. If $\Lambda$ is an $N$-set in $X_{h}$ such that the inverse of every point $\xi \in \Lambda$ is degenerate, then $T^{-1}(\Lambda)=N$ is an $N$-set in $X$ and $k(N)=T^{-1}[k(\Lambda)]$.

Proof. Let $\alpha$ and $\beta$ be distinct points in $\Lambda$ whose inverses are $a$ and $b$ respectively. By 3.15, $a \sim b$. It follows by 2.11 that there exists a unique $N$-set, $N \subset X$, which contains $a+b$. By the coherence and completeness of $N$, $T^{-1}(\Lambda) \subset N$.

If $y$ is an arbitrary point of $N$, it will be shown that the image $T(y)=\eta$ is in $\Lambda$. If this were false, then by 2.10 one of $\alpha \sim \eta$ or $\beta \sim \eta$ would be false. If some point $\xi$ in $X_{h}$ separates $\alpha$ from $\eta$, then $T^{-1}(\xi)$ is closed; moreover, if $S_{a}$ and $S_{y}$ denote the components of $X-T^{-1}(\xi)$ containing $a$ and $y$, respectively, $S_{a} \cdot S_{y}=0$. Otherwise, $S_{a}=S_{y}$. Then, by 3.7, $T\left(S_{a}\right)$ is connected, contained in $X_{h}-\xi$, and contains $\alpha+\eta$. This contradicts the separation of $\alpha$ and $\eta$ by the point $\xi$. Since $a \sim y$, it follows by 2.2 that $T^{-1}(\xi)$ is nondegenerate. But then $T^{-1}(\xi)=k(M)$ for some true cyclic element $M$. Three cases arise: (1) $N \cdot M=0$, (2) $N \cdot M$ is a single point $x$, and (3) $N \equiv M$ (see 2.12 and 2.15).

Cases (1) and (2). In either case the set $N-N \cdot M$ is contained in a single component $S$ of $X-N \cdot M(2.9)$. Thus $a+y \subset N \subset \bar{S} \subset X-k(M)$. But $T(\bar{S})$ is connected (3.7) and contained in $X_{h}-T[\dot{k}(M)]=X_{h}-\xi$. This contradicts the separation of $\alpha$ and $\eta$ by the point $\xi$.

Case (3). If $N \equiv M$, then $T^{-1}(\xi)=k(M)$ and 3.15 together imply that $T^{-1}(\alpha)=a \in M-k(M)$. Hence, by $3.14, \xi \sim \alpha$. Similarly, $\xi \sim \beta$. But then $\xi \in \Lambda$ and $T^{-1}(\xi)$ is nondegenerate which contradicts the hypothesis.

It has been established that $T^{-1}(\Lambda)=N$. It remains to show that $T^{-1}[k(\Lambda)]=k(N)$.

Let $\xi$ be any point in $k(\Lambda)$ and set $x=T^{-1}(\xi)$. If $x \notin k(N)$, then for some component $S$ of $X-N, x \in F(S)$. Since $N$ fulfills the hypotheses of the set $E$ of 3.11 , the component $S$ is an inverse set and $T(S)$ is a component of $X_{h}-T(N)=X_{h}-\Lambda$. If $\Gamma$ is any open set containing $\xi=T(x)$, the set $T^{-1}(\Gamma)$ is open and contains $x$. But then $x \in F(S)$ implies $\Gamma \cdot T(S) \neq 0$ and thus $\xi \in F[T(S)]$ contrary to $\xi \in k(\Lambda)$. Thus $x \in k(N)$, and $T^{-1}[k(\Lambda)] \subset k(N)$.

Finally, $k(N) \subset T^{-1}[k(\Lambda)]$. To see this, let $x \in k(N)$ and suppose that $T(x)=\xi \notin k(\Lambda)$. Since $\xi \in \Lambda$, there is some component $\Sigma$ of $X_{h}-\Lambda$ such that $\xi \in F(\Sigma)$. By 3.12, $T^{-1}(\Sigma)$ is a component of $X-N$. But $x \in k(N)$ implies $x \notin F\left[T^{-1}(\Sigma)\right]$; therefore $x \notin \overline{T^{-1}(\Sigma)}$. Moreover, by $3.10, \overline{T^{-1}(\Sigma)}$ is an inverse 
set so $X-\overline{T^{-1}(\Sigma)}$ is an open inverse set containing $x$. Thus the open set $T\left[X-\overline{T^{-1}(\Sigma)}\right]$ contains the point $\xi$ and is disjoint of $\Sigma$; this contradicts $\xi \in F(\Sigma)$.

The lemma follows.

\subsection{TheOREM. The hyperspace $X_{h}$ contains no true cyclic elements.}

Proof. Consider any $N$-set, $\Lambda \subset X_{h}$. There are two cases.

Case (1). If there exists a point $\alpha \in \Lambda$ such that $T^{-1}(\alpha)$ is nondegenerate, it will be shown that $\alpha \supset k(\Lambda)$ and so $\Lambda$ cannot be a true cyclic element (2.15). Now $T^{-1}(\alpha)=k(M)$ for some true cyclic element $M$. By 3.15 for any point $\xi \neq \alpha$ contained in $\Lambda, T^{-1}(\xi)$ is a point $x=F(S)$ for some component $S$ of $X-M$. By 3.11, $T(S)$ is a component of $X_{h}-\Lambda$ and $\xi \in F[T(S)]$. Thus $\alpha \supset k(\Lambda)$.

Case (2). For every point $\alpha \in \Lambda, T^{-1}(\alpha)$ is degenerate. By 3.16, $T^{-1}(\Lambda)=N$ is an $N$-set and $T^{-1}[k(\Lambda)]=k(N)$. If $N$ is a true cyclic element in $X$ or if $k(N)$ is degenerate, then $k(\Lambda)$ is a single point. In these cases $\Lambda$ is not a true cyclic element (2.15). If $N$ is not a true cyclic element but $k(N)$ is nondegenerate, then for some component $S$ of $X-N$ the frontier $F(S)$ contains $x$ and $y$ where $x \neq y$. By 3.11, the set $T(S)=\Sigma$ is a component of $X_{h}-\Lambda$. Also, $x$ and $y$ are distinct inverse sets. This implies that $F(\Sigma)$ is nondegenerate. By 2.15, $\Lambda$ is not a true cyclic element and the theorem is established.

3.18. In 2.1 the space $X$ was assumed to be connected. That this restriction imposed no loss of generality is easy to see. If the space $X$ is not connected, the definitions in 2.2 and 2.3 of separation and conjugacy need only be regarded relative to the individual components $X^{*}$ of the space $X$. Thus, a point $p$ will be said to separate two points $a$ and $b$ if (1) the points $a, b$, and $p$ lie in a single component $X^{*}$ of $X$, (2) $p=p$, and (3) $a$ and $b$ lie in distinct components of $X^{*}-p$. Similarly, two points $a$ and $b$ are conjugate if they lie in the same component $X^{*}$ of $X$ and are separated by no point of $X^{*}$.

The hyperspace $X_{h}$ of $X$ will be the totality of cyclic elements of all thecomponents of $X$, each being topologized according to 3.2.

A locally connected topological space is said to be acyclic if its hyperspace is topologically equivalent to itself. If a space has no true cyclic elements, then it is easily seen to be acyclic; conversely, if a space is acyclic, it has no true cyclic elements.

THEOREM. If $X$ is a locally connected topological space, then each true cyclic element of $X$ and the hyperspace $X_{h}$ are locally connected topological spaces. Moreover, the hyperspace $X_{h}$ is acyclic.

Proof. The proof is a direct consequence of $2.29,3.8,3.17$, and the remarks above. 


\section{Hereditary Classes OF SPACES}

4.1. The theorem of 3.18 suggests the following definition. A subclass $\mathfrak{H C}$ of the class $X$ of all locally connected topological spaces will be called hereditary if whenever $X$ is in $\mathfrak{H}$, the hyperspace $X_{h}$ is in $\mathfrak{H C}$ and each true cyclic element of $X$ is a member of $\mathfrak{F}$. The theorem of 3.18 can now be phrased as:

The totality of locally connected topological spaces is a hereditary class of spaces.

Remark. Any hereditary class is cyclicly reducible (Kuratowski and Whyburn [4]).

Consider the class $P$ of all peano spaces. The example consisting of two tangent circles in the plane is a member of the class. However, the hyperspace for this example contains only three points. Since such a space cannot be connected and metric, it is not a peano space. Thus, the class $P$ is not a hereditary class. On the other hand, every peano space is a locally connected topological space; therefore there exists a hereditary class which contains $P$..An immediate question is: what is the smallest class (if it exists) which is hereditary and which contains all peano spaces? This question is answered in 4.3 and 4.4.

4.2. TheOREM. The logical product or sum of any number of hereditary classes is a hereditary class.

The proof is immediate from the definitions.

4.3. Let $\mathbb{P}^{*}$ be the product of all the hereditary classes which contain the class $P$ of all peano spaces. One immediately obtains the

THEOREM. The class $\mathbb{P}^{*}$ is the smallest hereditary class which contains all peano spaces.

This result demonstrates the existence of an answer to the question posed in 4.1. A constructive definition of the class $P^{*}$ is found in the following section.

4.4. Theorem. A necessary and sufficient condition that a space $X$ be contained in the class $\mathcal{P}^{*}$ is that $X$ be either a peano space or the hyperspace of a peano space.

Proof. Let $P$ and $h[P]$ denote, respectively, the class of all peano spaces and the class of all their hyperspaces. The class $P+h[P]$ is hereditary by 3.18 and because every true cyclic element of a peano space is in $P$. Then by the definition of $\mathscr{P}^{*}$ one has $\mathbb{P}^{*} \subset \mathcal{P}+h[\mathcal{P}]$. The reverse inclusion follows since any hereditary class that contains $P$ must contain $h[\mathcal{P}]$.

4.5. The class $P$ may be defined as the totality of compact, metric spaces which are connected and locally connected. It would be interesting to know 
an analogous intrinsic definition for the class $\mathbb{P}^{*}$. With this in view, the remainder of the paper is devoted to a list of intrinsic properties of $\mathcal{P}^{*}$.

4.6. A subclass of $\mathcal{X}$ is invariant with respect to strongly continuous transformations if every strongly continuous image of a member of the class is again a member of the class (3.4).

Theorem. Any invariant subclass of $X$ is hereditary.

Proof. As the hyperspace $X_{h}$ of a space $X$ is a strongly continuous image of $X$ (see 3.5), half of the theorem is proved. For the last part we need the

Theorem. A true cyclic element of $X$ is a strongly continuous image of $X$.

Proof. Let $M$ denote any true cyclic element in the space $X$. Define the transformation $f(X)=M$ by

$$
f(x)=\left\{\begin{array}{l}
x \text { if the point } x \text { is in } M, \\
F(S) \text { if the point } x \text { is in the component } S \text { of } X-M .
\end{array}\right.
$$

Let $A$ be any open set in $M$; then $f^{-1}(A)=A^{*}$; and $A^{*}$ is open (2.22 and 2.24) On the other hand if $A$ is a set in $M$ such that $f^{-1}(A)=A^{*}$ is open in $X$, then $A$ is clearly open in $M$ because $A=M \cdot A^{*}$. Thus $f(X)=M$ is strongly continuous. ( $M$ is, in fact, a retract, see Borsuk [10].)

4.7. The reader will have no difficulty in proving that these subclasses of $X$ are invariant and hence hereditary (4.6):

(1) the class of connected spaces,

(2) the class of separable spaces,

(3) the class of compact spaces (for any of the usual interpretations of compactness),

(4) the class of spaces having the property that the number of components of an open set is denumerable,

(5) the class of spaces which are strongly continuous images of some fixed space.

An interesting subcase of (5) occurs when the fixed space is the closed unit interval. In this case the class is a subclass of the four preceding classes.

A tabulation of hereditary classes might be continued on the above lines. However, there are hereditary classes which are not invariant and some of these will be considered in the concluding sections of the paper.

4.8. Before turning to this topic some remarks should be made about the following separation axiom, attributed to Kolmogoroff (Alexandroff and Hopf $[1$, p. 58]).

\section{$T_{0}$ : If $x$ and $y$ are distinct points, then at least one of them is contained} in an open set that does not contain the other.

This axiom will be referred to as the $T_{0}$-property and topological spaces in which it is satisfied will be called $T_{0}$-spaces. 
If $x$ and $y$ are distinct points of a $T_{0}$-space such that $x \in \bar{y}$, then $x$ will be called a satellite of $y$.

Clearly, if $x$ is a satellite of $y$, then $y$ cannot be a satellite of $x$. This follows from the hypothesis $x \in \bar{y}$ and the $T_{0}$-property which then requires $y \cdot \bar{x}=0$. Thus one has the

THEOREM. In a $T_{0}$-space, the relation ' $x$ is a satellite of $y$ ' is asymmetric.

4.9. It will be shown in 4.10 that the class of locally connected $T_{0}$-spaces is hereditary. This result requires the

Lemma. If $x$ is a point in any locally connected topological space, then the set $\bar{x}$ is coherent (see 2.7.)

Proof. The lemma is trivial if $\bar{x}=x$. Suppose that for $y \neq x$, it is true that $y \in \bar{x}$, and $z$ is a point distinct from $x$ and $y$ and such that $\bar{z}=z$. If $S$ denotes the component of $X-z$ containing $x$, then $x+y$ is contained in $S$ since $x+y$ is connected (1.6). Thus $y \sim x$. The lemma follows by 2.5 .

CoROLlaRy. If a point $x$ in any locally connected topological space is an inverse set, then so is $\bar{x}$ an inverse set (see 3.2.)

Proof. Let $M$ be any true cyclic element and suppose that $\bar{x} \cdot k(M) \neq 0$. It follows by the coherence of $\bar{x}$ and the corollary of 2.20 that $x \in M$. But $x$ is an inverse set so $x \in M-k(M)$. Thus, by 2.19 , it is true that $\bar{x}=x$, and the theorem follows. If $\bar{x} \cdot k(M)=0$, then $\bar{x}$ is an inverse set by 3.10 .

4.10. TheOREM. The class of all locally connected $T_{0}$-spaces is hereditary.

Proof. Let $X$ be any space in the class $X$; suppose the $T_{0}$-property satisfied in $X$. Let $\xi$ and $\eta$ be distinct points in the hyperspace $X_{h}$. There are two cases according as (1) at least one of the inverses $T^{-1}(\xi)$ and $T^{-1}(\eta)$ is nondegenerate, or (2) both inverses are degenerate.

Case 1. Suppose $T^{-1}(\xi)=k(M)$ for some true cyclic element $M$. If $M \cdot T^{-1}(\eta)=0$, let $S$ be the component of $X-M$ which contains $T^{-1}(\eta)$; $S$ exists by 2.29 . Now $S$ is an open inverse set so $T(S)$ is an open set containing $\eta$ but not $\xi$ (3.11). If $M \cdot T^{-1}(\eta) \neq 0$, then $T^{-1}(\eta)=y \in M-k(M)$ by 2.12 and 2.15. Let $S$ be the component of $X-y$ containing $k(M)$ (2.28). Since $\bar{y}=y$, it follows that $S$ is open and $T(S)$ is an open set containing $\xi$ but not $\eta$ (3.11).

Case 2. Let $x=T^{-1}(\xi)$ and $y=T^{-1}(\eta)$. By the $T_{0}$-property in $x$, either $y \notin \bar{x}$ or $x \notin \bar{y}$; assume the former. By 4.9, $\bar{x}$ is an inverse set. Hence 3.11 may be applied as in Case 1 to obtain an open set containing $\eta$ but not $\xi$.

Remark. The example of 4.1 shows that the class of all locally connected $T_{1}$-spaces is not hereditary (Alexandroff and Hopf $[1$, p. 58]).

4.11. The hereditary classes discussed so far have been of a general nature. 
In those to follow, attention is focused more explicitly on certain properties of peano spaces.

If $M$ is any true cyclic element in a peano space, then the kernel of $M$ is such that its closure contains $M$. Let $\mathfrak{H C}_{1}$ be the totality of spaces in $\mathcal{X}$ for which $\overline{k(N)}=N$ for any $N$-set.

TheOREM. The class $\mathfrak{H C}_{1}$ is hereditary.

Proof. Let $X$ be any space in the class $\mathfrak{F C}_{1}$ and let $\Lambda$ be any $N$-set in $X_{h}$. There are two cases.

Case 1. If every point in $\Lambda$ has a degenerate inverse, the result follows from 3.16 and the hypothesis that $\overline{k(N)}=N$ in $X$.

Case 2. Suppose that some point $\xi \in \Lambda$ has a nondegenerate inverse $k(M)$, where $M$ is a true cyclic element in $X$. By $3.15, T^{-1}(\Lambda) \subset M$. By the continuity of $T$ and the hypothesis, $\overline{k(N)}=N$, it is true that $T(M) \subset \Lambda$. Hence $M$ is the inverse of $\Lambda$. It follows (3.11) that $k(\Lambda)=\xi$ and $\bar{\xi}=\Lambda$.

4.12. Lemma. If $X$ is in $\mathfrak{F}_{1}$, then for every point $\xi$ in $X_{h}$, one has $T^{-1}(\bar{\xi})$ $=\overline{T^{-1}(\xi)}$.

Proof. Clearly $T^{-1}(\bar{\xi}) \supset T^{-1}(\xi)$ and hence

$$
T^{-1} \overline{(\xi)}=\overline{T^{-1}(\bar{\xi})} \supset \overline{T^{-1}(\xi)}
$$

To see the reverse inclusion, consider first the case in which $T^{-1}(\xi)=x$, a single point. Since $x$ is an inverse set, the same is true of $\bar{x}(4.9)$. Thus if any point $y$ is not in $\bar{x}=\overline{T^{-1}(\xi)}$, then $y$ is in some component $S$ of $X-\bar{x}$. By the corollary of $4.9, \bar{x}$ satisfies the hypotheses of 3.11. Hence $S$ is an inverse set and $T(S)$ is a component of $X_{h}-T(\bar{x}) \subset X_{h}-\xi$. Thus there is an open set, $T(S)$, containing $T(y)$ but not $\xi$. Hence $T(y) \notin \bar{\xi}$. Therefore $T^{-1}(\bar{\xi}) \subset \overline{T^{-1}(\xi)}$. In case $T^{-1}(\xi)=k(M)$ for some true cyclic element $M$, it follows that $T^{-1}(\bar{\xi})=M=\overline{k(M)}=\overline{T^{-1}(\xi)}$ and the lemma is proved.

4.13. CoROllary. Under the hypotheses of the lemma, if $\xi$ and $\eta$ are distinct points in the hyperspace $X_{h}$ of $X$, then $\xi \in \bar{\eta}$ if and only if either: (1) $T^{-1}(\xi)$ and $T^{-1}(\eta)$ are single points such that $T^{-1}(\xi) \in \overline{T^{-1}(\eta)}$, or $(2) T^{-1}(\eta)=k(M)$ for some true cyclic element $M$, and $T^{-1}(\xi)=x \in M-k(M)$.

The proof is easy and will be left to the reader.

4.14. Let $\mathfrak{H}_{2}$ be the class of all spaces $X$ in $\mathfrak{F}_{1}$ having the property that if $x$ is any point, the frontier of any component of $X-\bar{x}$ is a single point.

Theorem. The class $\mathfrak{H}_{2}$ is hereditary.

Proof. Consider $X \in \mathfrak{F C}_{2}$. If $M$ is a true cyclic element in $X$ it certainly has the property $(2.25)$. Let $\xi$ be any point in $X_{h}$. If $\bar{\xi}=\xi$, the result is im- 
mediate. Suppose $\bar{\xi} \neq \xi$. By 4.12, $T^{-1}(\bar{\xi})=\overline{T^{-1}(\xi)}$. If $T^{-1}(\xi)$ is degenerate, then any component of $X-\overline{T^{-1}(\xi)}$ has a single frontier point by hypothesis. If $T^{-1}(\xi)=k(M)$ for some true cyclic element, then $M=\overline{T^{-1}(\xi)}$ and again any component of $X-\overline{T^{-1}(\xi)}$ has a single frontier point. It follows easily with the help of 3.11 and 3.12 that every component of $X_{h}-\bar{\xi}$ has a single frontier point.

4.15. Let $\mathfrak{F C}_{3}$ be the totality of spaces $X$ in $\mathfrak{H C}_{2}$ such that if $N$ is any $N$-set, then the frontier of any component of $X-N$ is a single point.

Theorem. The class $\mathfrak{H}_{3}$ is hereditary.

Proof. Suppose $X \in \mathfrak{H}_{3}$. Let $\Lambda$ be any $N$-set in $X_{h}$, and let $\Sigma$ be any component of $X_{h}-\Lambda$. If for some point $\xi \in \Lambda$, it is true that $T^{-1}(\xi)=k(M)$ for some true cyclic element $M$, then $\overline{T^{-1}(\xi)}=M$ and $\bar{\xi}=\Lambda$. The frontier $F(\Sigma)$ is a single point by 4.14 . Suppose that for every point $\xi \in \Lambda$ the set $T^{-1}(\xi)$ is degenerate. By 3.16, $T^{-1}(\Lambda)=N$ is an $N$-set and $k(N)=T^{-1}[k(\Lambda)]$. By the assumption on the inverses of points in $\Lambda$, the set $N$ is not a true cyclic element. It follows by 2.15 that the kernel $k(N)$ is degenerate. But $\overline{k(N)}=N$ and hence $N$ is of the form $\bar{x}$ where $x$ is a single point. Then $F(\Sigma)$ is a single point by 4.14 .

4.16. TheOREM. Let $X$ be in $\mathfrak{F C}_{2}$. If $x$ is any point in $X$ such that $\bar{x} \neq x$, then the set $\bar{x}$ is an $N$-set.

Proof. The set $\bar{x}$ was proved coherent in 4.9. Let $y$ be any point in $X-\bar{x}$. Let $S$ denote the component of $X-\bar{x}$ that contains $y$ and let $R$ denote the sum of the remaining components of $X-\bar{x}$. As in the proof of 2.19 , one shows that the point $F(S)$ separates $y$ from every point in $\bar{x}-y$. It follows that $\bar{x}$ is complete and the theorem is proved.

Corollary. Let $X$ be any $T_{0}$-space in $\mathfrak{H C}_{2}$. If the point $x \in X$ is a satellite of the point $y$, then $y$ is not a satellite of any point.

Proof. If $x \in \bar{y}$ and $y \in \bar{z}$, then $z \neq x$ since $y \cdot \bar{x}=0$. Moreover, $z \cdot \bar{y}=0$. Let $S$ be the component of $X-\bar{y}$ that contains $z$. Now $\bar{z} \subset \bar{S}=S+F(S)$ and $F(S)$ is a single closed point. Thus $F(S) \neq y$ since $\bar{y} \neq y$. But then $y \cdot \bar{z}=0$ which is contradictory.

4.17. Conclusion. Let $\Upsilon$ denote the totality of all locally connected topological spaces which are (1) strongly continuous images of the closed unit line interval, (2) $T_{0}$-spaces in $\mathfrak{H C}_{3}$. By the theorem of $4.2, \Upsilon$ is a hereditary class. Moreover, it contains the class $P^{*}$ (see 4.3). More restrictive hereditary classes which contain $\mathcal{P}^{*}$ will suggest themselves to the reader. The class $\Upsilon$ has been mentioned because it yields considerable insight into the nature of the hyperspaces of peano spaces.

It is a consequence of 2.15 and 4.15 that in any space of the class $\Upsilon$ every $N$-set is either a true cyclic element or a set of the form $\bar{x}$ where $x$ is a point. Thus, in any peano hyperspace, the only $N$-sets are of the form $\bar{\xi}$ where $\xi$ is 
a point. The corollary of 4.13 states explicitly how such $N$-sets arise; namely, from the incidence in the original peano space of a degenerate cyclic element on a true cyclic element. This relationship is certainly not symmetrical. Therefore, the asymmetry of the $T_{0}$-property seems particularly fitting in this connection.

\section{BIBLIOGRAPHY}

1. P. Alexandroff and H. Hopf, Topologie I, Berlin, 1935.

2. F. Hausdorff, Mengenlehre, Berlin, 1927 or 1935.

3. B. Knaster and C. Kuratowski, Sur les ensembles connexes, Fundamenta Mathematicae, vol. 2 (1921), p. 206.

4. C. Kuratowski and G. T. Whyburn, Sur les éléments cycliques et leurs applications, Fundamenta Mathematicae, vol. 16 (1930), p. 305.

5. G. T. Whyburn, Cyclicly connected continuous curves, Proceedings of the National Academy of Sciences, vol. 13 (1927), p. 31.

6. - Concerning the structure of a continuous curve, American Journal of Mathematics, vol. 50 (1928), p. 167.

7. R. L. Moore, Rice Institute Pamphlet, vol. 23, no. 1, 1936.

8. T. Rad6 and P. V. Reichelderfer, Cyclic transitivity, Duke Mathematical Journal, vol. 6 (1940), p. 474.

9. J. W. T. Youngs, Arc-spaces, Duke Mathematical Journal, vol. 7 (1940), p. 68.

10. K. Borsuk, Sur les rétractes, Fundamenta Mathematicae, vol. 27 (1931), p. 152.

Ohio State University,

Columbus, Оhio

Purdue University,

LAFAYETTE, IND. 